Pacific

Journal of

Mathematics

\title{
SIMPLY CONNECTED
}

NONPOSITIVELY CURVED SURFACES IN $\mathbb{R}^{3}$

HSUNGROW CHAN

Volume $223 \quad$ No. 1

January 2006 


\title{
SIMPLY CONNECTED NONPOSITIVELY CURVED SURFACES IN $\mathbb{R}^{3}$
}

\author{
HSUNGROW CHAN
}

\begin{abstract}
We consider complete noncompact simply connected nonpositively curved surfaces that are twice continuously differentiably embedded in Euclidean three space. If such a surface has square integrable second fundamental form, then it must be a plane.
\end{abstract}

\section{Introduction}

The study of minimal surfaces has a long tradition and has been extended in many directions: surfaces of constant mean curvature, isometric immersion of surfaces into hyperbolic spaces or other higher dimensional spaces, and so on. Here, we try to go in another direction. Consider the condition

$$
\int|B|^{2} d a<\infty
$$

where $|B|$ is the length of second fundamental form. Now, $|B|^{2}=4|H|^{2}-2 K$, where $H$ is the mean curvature and $K$ is the Gauss curvature. The Gauss curvature of minimal surfaces is nonpositive. We examine what minimal surface results continue to hold if we extend the minimal condition $H \equiv 0$ to $K \leq 0$ but require (1) instead. We recall:

Theorem [Xavier 2001]. Let $M \subset \mathbb{R}^{3}$ be a complete simply connected embedded minimal surface whose Gaussian curvature is bounded from below. If there is a plane whose intersection with $M$ is transversal and connected then $M$ is a plane or a helicoid.

Theorem [Meeks and Rosenberg 2005]. A properly embedded simply connected minimal surface in $\mathbb{R}^{3}$ is either a plane or a helicoid.

Minimal surfaces can be divided into two classes: those of finite total curvature and those of infinite total curvature. For the case of finite total curvature we have $\int|B|^{2}=-2 \int K<\infty$, so condition (1) is satisfied. In this paper we prove:

MSC2000: 53A05, 49Q05.

Keywords: simply connected, nonpositively curved surfaces, minimal surfaces.

This work was partially supported by the National Science Council and the National Center for Theoretical Sciences (NCTS), Taiwan. 
Theorem 1. A complete simply connected embedded $C^{2}$-surface $M$ in $\mathbb{R}^{3}$ with $K \leq 0$ and (1) is a plane.

We can apply the proof of Theorem 1 to recover the finite total curvature case of Meeks and Rosenberg's Theorem.

S. Bernstein [1915-17] proved that if $M$ is an entire minimal graph, then $M$ must be a plane. This theorem has been generalized; see [Fujimoto 1988; Osserman 1971; Xavier 1981]. In [Chan 2000], we showed:

Theorem 2. Suppose $M$ is a complete, oriented, one-ended, nonpositively curved Riemannian surface with an isolated set of parabolic points $\{p \in M: K(p)=0\}$. Then $M$ cannot be $C^{2}$ isometrically immersed in $\mathbb{R}^{3}$ with (1) and one embedded end.

The stipulation of one embedded end cannot be weakened, because of the catenoid and Enneper's surface. The conditions in Theorem 2, apart from the isolatedness of parabolic points, are satisfied by the following example. Let $(x, y, z)$ be the usual coordinates of $\mathbb{R}^{3}$. Let $S$ be the locus of points satisfying

$$
(1-z)\left(y^{2}+z(1+z)\right)=(1+z)\left(x^{2}-z(1-z)\right) .
$$

By the Implicit Function Theorem, $S$ is a surface in $\mathbb{R}^{3}$. In fact, $S$ lies in the region $|z| \leq 1$ and is not simply connected (its genus is 1). In [Chan and Treibergs 2001], we improved our theorem as follows:

Theorem 3. Suppose $M$ is a complete, oriented, nonpositively curved Riemannian surface $C^{2}$ isometrically immersed in $\mathbb{R}^{3}$ satisfying (1) and having one embedded end near infinity. Then $M$ must lie in a slab (a region between two parallel planes).

Since the plane stays in the slab, this generalizes Bernstein's Theorem. In that paper, we also provided explicit examples of nonpositively curved surfaces of genus $n$. In cylindrical coordinated of $\mathbb{R}^{3}$, for $n \geq 2$ an integer, the surface

$$
r^{n}(z-\cos n \theta)=z-z^{3}
$$

has genus $n-1$ and lies in the slab $|z| \leq 1$. Thus, simple connectivity is a critical condition for the Theorem 1.

\section{Proof of Theorem 1.}

Morse's theorems relate the topology of manifolds to the singularities of Morse functions on them. In this section, we apply Morse theory to noncompact complete surfaces in $\mathbb{R}^{3}$ by finding Morse functions that have no critical points outside a large compact set. 
Let $M$ be a complete, oriented, connected, nonpositively curved surface $C^{2}$ immersed in $\mathbb{R}^{3}$. White [1987] proved that if (1) is satisfied, the Gauss map extends continuously to one point near infinity and $M$ is properly immersed near infinity.

The simple connectivity and embeddedness of the surface $M$ in Theorem 1 imply that $M$ has one embedded end. Let $p$ denote the point at infinity of the surface. By White's theorem, (1) implies that the Gauss image $g(p)$ is one point and $M$ is properly embedded. Then there exists a punctured disk $U_{\epsilon}(p)$ that is embedded and such that $|g(x)-g(p)|<\epsilon$ for all $x \in U_{\epsilon}(p)$. The outside of a big compact set of $M$ can be considered as a graph of a function over the $x y$-plane $P$.

We divide the proofs into two cases, $K \equiv 0$ and $K \not \equiv 0$. Assume that $K \equiv 0$ on $M$ in Theorem 1. According to [Hartman and Nirenberg 1959], $M$ is a generalized cylinder. By (1), $M$ is a plane. Assume instead that $K \not \equiv 0$. Then there is a point $p_{0}$ such that $K\left(p_{0}\right)<0$. We may assume that the tangent plane $T_{p_{0}} M$ is not parallel to $P$ and that the distance function to $T_{p_{0}} M$ (the height function above $T_{p_{0}} M$ ) is a Morse function. Since the normal directions of a neighborhood of $p_{0}$ form a set of full measure on the unit sphere, there is a point $p_{1}$ in a small neighborhood of $p_{0}$ such that the distance function of the tangent plane $T_{p_{1}} M$ is a Morse function and $T_{p_{1}} M$ is not parallel to $P$. Then $p_{1}$ is a critical point of $f$. Because the curvature is nonpositive at the critical points, all indices are 1 .

For a Morse function on a compact surface $\bar{M}$, Morse's formula [Milnor 1963] says that the Euler characteristic is the algebraic sum of the numbers $C_{\lambda}$ of critical points of index $\lambda$ :

$$
\chi(\bar{M})=\sum_{\lambda}(-1)^{\lambda} C_{\lambda}
$$

In the case of a surface, $\lambda$ takes the values 0,1 , and 2 , so $\chi(\bar{M})=C_{0}-C_{1}+C_{2}$.

To prove Theorem 1, we need to modify Morse's formula. Let $f$ be the distance function from $T_{p_{1}} M$ on $M$. Choose $\epsilon$ so small that $\left|g\left(p_{1}\right)-g(p)\right|>\epsilon$. Then no critical points of $f$ occur in $U_{\epsilon}(p)$. Because the Gauss map extends continuously to $p$, the point at infinity, and because $M$ is the graph of a function in $U_{\epsilon}(p)$, we can cut out a disk about $p$ in $U_{\epsilon}$ and glue back a spherical cap containing exactly two new critical points of $f$, of indices 0 and 2 respectively. Call the new surface $\widehat{M}$. After gluing, $\chi(\widehat{M})=\chi(M)+1$. Thus the Morse formula for noncompact surfaces yields

$$
\chi(M)=\chi(\widehat{M})-1=1-C_{1}+1-1=1-C_{1} .
$$

There is at least one critical point $p_{1}$ whose index is 1 for $f$, so $\chi(M)$ has a upper bound of zero, contradicting simple connectivity. Thus $M$ is a plane. This completes the proof. 


\section{Acknowledgments}

I thank Andrejs Treibergs for his advice, which shortened the proof.

\section{References}

[Bernstein 1915-17] S. Bernstein, "Sur un théoréme de géométrie et ses applications aux équations aux dérivées partielles du type elliptique", Comm. Soc. Math. Kharkov 15 (1915-17), 38-45. JFM 48.1401.01

[Chan 2000] H. Chan, "Nonexistence of nonpositively curved surfaces with one embedded end", Manuscripta Math. 102:2 (2000), 177-186. MR 2001f:53069 Zbl 0967.53038

[Chan and Treibergs 2001] H. Chan and A. Treibergs, "Nonpositively curved surfaces in $\mathbf{R}^{3}$ ", $J$. Differential Geom. 57:3 (2001), 389-407. MR 2003d:53010 Zbl 1041.53001

[Fujimoto 1988] H. Fujimoto, "On the number of exceptional values of the Gauss maps of minimal surfaces”, J. Math. Soc. Japan 40:2 (1988), 235-247. MR 89b:53013 Zbl 0629.53011

[Hartman and Nirenberg 1959] P. Hartman and L. Nirenberg, "On spherical image maps whose Jacobians do not change sign”, Amer. J. Math. 81 (1959), 901-920. MR 23 \#A4106 Zbl 0094.16303

[Meeks and Rosenberg 2005] W. H. Meeks, III and H. Rosenberg, "The uniqueness of the helicoid", Ann. of Math. (2) 161:2 (2005), 727-758. MR 2153399

[Milnor 1963] J. Milnor, Morse theory, Annals of Mathematics Studies 51, Princeton University Press, Princeton, N.J., 1963. MR 29 \#634 Zbl 0108.10401

[Osserman 1971] R. Osserman, "The convex hull property of immersed manifolds", J. Differential Geometry 6 (1971), 267-270. MR 45 \#7647 Zbl 0226.53009

[White 1987] B. White, "Complete surfaces of finite total curvature", J. Differential Geom. 26:2 (1987), 315-326. MR 88m:53020 Zbl 0631.53007

[Xavier 1981] F. Xavier, "The Gauss map of a complete nonflat minimal surface cannot omit 7 points of the sphere", Ann. of Math. (2) 113:1 (1981), 211-214. MR 82b:53015 Zbl 0477.53007

[Xavier 2001] F. Xavier, "Embedded, simply connected, minimal surfaces with bounded curvature", Geom. Funct. Anal. 11:6 (2001), 1344-1356. MR 2003c:53019 Zbl 1004.53007

Received March 7, 2004. Revised December 24, 2004.

HSUNGROW CHAN

NATional Pingtung University of EdUCATION

Pingtung, 900-03 TAIWAN

hchan@mail.npttc.edu.tw 